\title{
An efficient algorithm for solving Fredholm integral equations using piecewise quadratic interpolation
}

\section{Azedine Rahmoune ${ }^{1 *}$, Boumedien Sehili $^{2}$ \\ ${ }^{1,2}$ Department of Mathematics, University of Bordj Bou Arréridj, 34030 El Anasser, Bordj Bou Arréridj, Algeria}

\section{ABSTRACT}

The main purpose of the present paper is to suggest a new simpler efficient algorithm based on piecewise interpolation techniques for solving Fredholm integral equations by using quadratic polynomials with Newton's divided differences approach. The proposed method is consistent and remains non sensitive even when the number of knots increases. The convergence theorems have been established. Also, numerical results to illustrate the efficiency of the method are presented.

Keywords: Piecewise quadratic polynomials, Integral equations, Newton's divided differences, Simpson's rule.

Corresponding Author: " Azedine Rahmoune, e-mail: a.rahmoune@univ-bba.dz

\section{INTRODUCTION}

We consider Fredholm integral equation of the second kind

$$
\mu \phi(s)-\int_{a}^{b} k(s, \tau) \phi(\tau) d \tau=f(s), a \leq s \leq b
$$

or in operator form,

$$
(\mu-K) \phi=f
$$

where $k(s, \tau), \phi(s), f(s)$ are assumed to be continuous and $\mu \neq 0$. The associated linear integral operator $K$ is compact from a Banach space $C[a, b]$ into itself. In practice, equation (1.1) occur naturally in a wide variety of physical applications which included radiative heat transfer problems, diffusion theory and particle transport problems. Also, it is well known that a boundary value problems for a differential equation can often be converted and condensed in a single Fredholm integral equation. A reader may consult Refs. [5,6,11] for more details. Fredholm integral equations have been extensively investigated theoretically and numerically in recent years by several authors, for instance see Refs. $[4,7,8,9,12]$. This paper is an extended version of work published in [8]. The advantage of this method is that it is more effective, and implementation algorithm is simple to achieve, programming need a few instructions using many kinds of computer software, including free software that is also open source. 


\section{PIECEWISE QUADRATIC APPROXIMATION}

In the field of numerical analysis, it is known that the polynomial interpolant is very sensitive to the choice of the control points. Of course, this problem can be solved by using the Chebyshev points for instance. However, this adjustment produce an approximation that, differs very little from the best one of the same order. This allows us to illustrate the essential limitation of polynomial approximation. If the function to be approximated is badly behaved locally in the interval of approximation, then the approximation is globally poor. This dependence can be achieved when using piecewise polynomial approximants.

For many years, piecewise polynomial approximation has been an active research area in applied mathematics, and has had impact on various applications, in constructing a curves, in computer graphics, in data fitting, and especially to obtain numerical integration and differentiation formulas.

We want to approximate $\phi(s)$ on an interval $[a, b]$ by using quadratic piecewise polynomials. Let $n \geq 2$ be an even integer. Define $h=(b-a) / n$ and $s_{j}=a+j h, j=0,1, \ldots, n$. Then, on each subinterval $I_{j}=\left[s_{j}, s_{j+2}\right], j$ even number from 0 to $n-2$, the quadratic polynomial passing through three given data points $\left(s_{j}, \phi_{j}\right),\left(s_{j+1}, \phi_{j+1}\right),\left(s_{j+2}, \phi_{j+2}\right)$, (for simplicity, we use the notation $\phi_{j}$ to denote $\phi\left(s_{j}\right)$ in Newton form can be constructed via a divided differences as follows

$$
\pi_{2, j}(s)=\phi_{j}+\frac{\Delta \phi_{j}}{h}\left(s-s_{j}\right)+\frac{\Delta^{2} \phi_{j}}{2 h^{2}}\left(s-s_{j}\right)\left(s-s_{j+1}\right),
$$

where

$$
\left(\begin{array}{c}
\phi_{j} \\
\Delta \phi_{j} \\
\Delta^{2} \phi_{j}
\end{array}\right)=\left(\begin{array}{ccc}
1 & 0 & 0 \\
-1 & 1 & 0 \\
1 & -2 & 1
\end{array}\right)\left(\begin{array}{c}
\phi_{j} \\
\phi_{j+1} \\
\phi_{j+2}
\end{array}\right)
$$

For the quadratic interpolation error, it is easy to show that

$$
\left|\phi(s)-\pi_{2, j}(s)\right| \leq M \frac{\sqrt{3}}{27} h^{3},
$$

where $M=\max _{s \in I_{j}}\left|\phi^{(3)}(s)\right|$.

\section{METHOD DESCRIPTION}

Our aim in this section is to develop a numerical discretization scheme for Fredholm integral equation of the second kind by interpolating the unknown solution using piecewise quadratic polynomials with Newton's divided differences. For convenience, we rewrite the given integral equation (1.1) in the following form 


$$
\mu \phi(s)-\sum_{\substack{j=0 \\ j \text { even }}}^{n-2} \int_{I_{j}} k(s, \tau) \phi(\tau) d \tau=f(s),
$$

According to (1.5) it follows that for $h$ sufficiently small, the unknown solution $\phi(\tau)$ can be approximated by quadratic polynomial $\pi_{2, j}(\tau)$ on each subinterval $I_{j}, j$ even from 0 to $n-2$, then

$$
\int_{s_{j}}^{s_{j+2}} k(s, \tau) \phi(\tau) d \tau \approx \int_{s_{j}}^{s_{j+2}} k(s, \tau) \pi_{2, j}(\tau) d \tau
$$

Next, substituting $\pi_{2, j}(\tau)$ by the expression given by (1.3). Therefore

$$
\int_{s_{j}}^{s_{j+2}} k(s, \tau) \pi_{2, j}(\tau) d \tau=\left(\alpha_{j}(s), \beta_{j}(s), \gamma_{j}(s)\right)\left(\begin{array}{c}
\phi_{j} \\
\Delta \phi_{j} \\
\Delta^{2} \phi_{j}
\end{array}\right)
$$

where

$$
\begin{gathered}
\alpha_{j}(s)=\int_{s_{j}}^{s_{j+2}} k(s, \tau) d \tau, \\
\beta_{j}(s)=\frac{1}{h} \int_{s_{j}}^{s_{j+2}} k(s, \tau)\left(\tau-s_{j}\right) d \tau, \\
\gamma_{j}(s)=\frac{1}{2 h^{2}} \int_{s_{j}}^{s_{j+2}} k(s, \tau)\left(\tau-s_{j}\right)\left(\tau-s_{j+1}\right) d \tau .
\end{gathered}
$$

These integral quantities must usually be evaluated numerically by using efficient quadrature rules. In such case, the use of Simpson's $1 / 3^{\text {rd }}$ rule would be quite sufficient. Then

$$
\begin{gathered}
\alpha_{j}(s) \approx a_{j}(s)=\frac{h}{3}\left[k\left(s, s_{j}\right)+4 k\left(s, s_{j+1}\right)+k\left(s, s_{j+2}\right)\right], \\
\beta_{j}(s) \approx b_{j}(s)=\frac{h}{3}\left[4 k\left(s, s_{j+1}\right)+2 k\left(s, s_{j+2}\right)\right], \\
\gamma_{j}(s) \approx c_{j}(s)=\frac{h}{3} k\left(s, s_{j+2}\right) .
\end{gathered}
$$

To organize and summarize our ideas with an approach global, for a given equidistant interpolatory subdivision, we can define a sequences of Newton piecewise interpolation operators $K_{n}: \phi \rightarrow K_{n} \phi($.$) as follow$

$$
K_{n} \phi(s)=W_{n}(s) \Delta \Phi
$$

where

$$
\begin{gathered}
W_{n}(s)=\left(a_{0}(s), b_{0}(s), c_{0}(s), a_{2}(s), b_{2}(s), \ldots, a_{n-2}(s), b_{n-2}(s), c_{n-2}(s)\right) \\
\Delta \Phi=\left(\phi_{0}, \Delta \phi_{0}, \Delta^{2} \phi_{0}, \phi_{2}, \Delta \phi_{2}, \Delta^{2} \phi_{2}, \ldots, \phi_{n-2}, \Delta \phi_{n-2}, \Delta^{2} \phi_{n-2}\right)^{T}
\end{gathered}
$$

Furthermore, with an explicit formula, we can write 


$$
\Delta \Phi=\mathrm{D} \Phi \text {, where } \mathrm{D}=\left(\begin{array}{ccccccc}
1 & 0 & 0 & 0 & \mathrm{~L} & 0 & 0 \\
-1 & 1 & 0 & 0 & \mathrm{~L} & 0 & 0 \\
1 & -2 & 1 & 0 & \mathrm{~L} & 0 & 0 \\
0 & 0 & 0 & \mathrm{O} & 0 & 0 & 0 \\
\mathrm{M} & \mathrm{M} & \mathrm{M} & 0 & 1 & 0 & 0 \\
0 & 0 & 0 & 0 & -1 & 1 & 0 \\
0 & 0 & 0 & 0 & 1 & -2 & 1
\end{array}\right), \Phi=\left(\begin{array}{c}
\phi_{0} \\
\phi_{1} \\
\phi_{2} \\
\mathrm{M} \\
\phi_{n-2} \\
\phi_{n-1} \\
\phi_{n}
\end{array}\right)
$$

So, to seek an approximate solution to the continuous problem (1.2), the idea will be to actually solve the semi-discrete problem

$$
\left(\mu-K_{n}\right) \phi_{n}(s)=f(s)
$$

in the sense that the residual part

$$
r(s)=\left(\mu-K_{n}\right) \phi_{n}(s)-f(s),
$$

is zero at the selected collocation points $s=s_{l}, l$ from 0 to $n$. Clearly, one is led to solving an algebraic system of the form

$$
\mu \Phi-\mathrm{Q} \Delta \Phi=\mathrm{F},
$$

In order to illustrate this method, for example with $n=4$, we try to interpolate the unknown solution using an equidistant subdivision of five knots $\left\{s_{j}, j=0, \ldots, 4\right\}$. The proposed discretization scheme lead to a matrix problem of the form (1.21) such that

and

$$
\begin{aligned}
\mathrm{Q} & =\left(\begin{array}{llllll}
a_{0}\left(s_{0}\right) & b_{0}\left(s_{0}\right) & c_{0}\left(s_{0}\right) & a_{2}\left(s_{0}\right) & b_{2}\left(s_{0}\right) & c_{2}\left(s_{0}\right) \\
a_{0}\left(s_{1}\right) & b_{0}\left(s_{1}\right) & c_{0}\left(s_{1}\right) & a_{2}\left(s_{1}\right) & b_{2}\left(s_{1}\right) & c_{2}\left(s_{1}\right) \\
a_{0}\left(s_{2}\right) & b_{0}\left(s_{2}\right) & c_{0}\left(s_{2}\right) & a_{2}\left(s_{2}\right) & b_{2}\left(s_{2}\right) & c_{2}\left(s_{2}\right) \\
a_{0}\left(s_{3}\right) & b_{0}\left(s_{3}\right) & c_{0}\left(s_{3}\right) & a_{2}\left(s_{3}\right) & b_{2}\left(s_{3}\right) & c_{2}\left(s_{3}\right) \\
a_{0}\left(s_{4}\right) & b_{0}\left(s_{4}\right) & c_{0}\left(s_{4}\right) & a_{2}\left(s_{4}\right) & b_{2}\left(s_{4}\right) & c_{2}\left(s_{4}\right)
\end{array}\right) \\
\Delta \Phi & =\left(\phi_{0}, \Delta \phi_{0}, \Delta^{2} \phi_{0}, \phi_{2}, \Delta \phi_{2}, \Delta^{2} \phi_{2}\right)^{T}, \Phi=\left(\phi_{0}, \phi_{1}, \phi_{2}, \phi_{3}, \phi_{4}\right)^{T},
\end{aligned}
$$

$$
\mathrm{F}=\left(f\left(s_{0}\right), \ldots, f\left(s_{4}\right)\right)^{T}
$$

Therefore, by using (1.18), the algebraic system (1.21) becomes

$$
\mathrm{A} \Phi=\mathrm{F}
$$

where $\mathrm{A}=\mu-\mathrm{QD}$.

Finally, we remark that, for the given equation (1.1) we obtain an algebraic system of the form (1.25) and the implementation of the suggested method requires the exhibition of a simple algorithm to perform the following instructions:

1. Compute the matrix $\mathrm{Q}$,

2. Generate the matrix denoted by $\mathrm{D}$,

3. Solve the resulting system $(\mu-\mathrm{QD}) \Phi=\mathrm{F}$. 
After determining the solution vector $\Phi$, it is easy to obtain the piecewise divided differences vector $\Delta \Phi$ by using (1.18). Consequently, the general solution to the equation (1.19) can be achieved at all other points $[a, b]$ by the following equation

$$
\phi_{n}(s)=\frac{1}{\mu}\left[f(s)+W_{n}(s) \Delta \Phi\right], a \leq s \leq b
$$

\section{CONVERGENCE ANALYSIS}

We will study convergence of the suggested method using the notion of collectively compact operator approximations, which was first introduced by Anselone, P. M. For more details, a complete presentation is given in his famous classical book [1].

Let $K: C[a, b] \rightarrow C[a, b]$ be a compact linear operator and $\mu-K$ be injective, it is well known that, if the following proprieties are satisfied.

(P1) The set $\{K: C[a, b] \rightarrow C[a, b]\}_{n \geq 1}$ of linear operators is collectively compact.

(P2) $K_{n} \phi \rightarrow K \phi, n \rightarrow \infty$ for all $\phi \in C[a, b]$.

Then for sufficiently large $n$, more precisely for all $n$ with

$$
\left\|(\mu-K)^{-1}\left(K_{n}-K\right) K_{n}\right\|<|\mu|,
$$

the inverse operators $\left(\mu-K_{n}\right)^{-1}$ exist and are bounded by

$$
\left\|\left(\mu-K_{n}\right)^{-1}\right\| \leq \frac{1+\left\|(\mu-K)^{-1} K_{n}\right\|}{|\mu|-\left\|(\mu-K)^{-1}\left(K_{n}-K\right) K_{n}\right\|} \leq C_{s},
$$

For the solution of the equations (1.2) and (1.19) we have the error estimate

$$
\left\|\phi-\phi_{n}\right\| \leq C_{s}\left\|\left(K_{n}-K\right) \phi\right\|
$$

where $C_{s}$, is a positive constant.

In our case, it is a simple matter to verify that the sequences of operators defined by (1.15) can be reduced to the form

$$
K_{n} \phi(s)=\sum_{j=0}^{n} w_{j}(s) \phi\left(s_{j}\right),
$$

In fact, it suffices to substitute $\Delta \Phi$ by DФ. Referring still to Anselone [1], it is also shown that the set of numerical quadrature operators of the form (1.30) satisfies the both proprieties (P1) and (P2).

Now, in order to examine the rate of convergence. According to Atkinson [4, pp.79] we have the following proposition

Proposition 1. Consider the sequence of Newton piecewise interpolation operators $A_{n}$ defined by (1.15). Further assume that $\phi \in C^{4}[a, b]$ and the kernel $k_{s}(\tau)=k(s, \tau) \in C^{1}[a, b]$.

Then for sufficiently large $n, K_{n} \phi \rightarrow K \phi$, more precisely we have

$$
\left(K_{n}-K\right) \phi(s)=O\left(h^{4}\right)
$$




\section{NUMERICAL EXPERIMENTS}

In this section, we applied the method presented in this paper for solving integral equation (1.1). In the Table1, $E_{\max }$ is the maximum absolute error at 1000 selected equally spaced points, defined by

$$
E_{\max }=\max _{j}\left|\phi\left(s_{j}\right)-\phi_{n}\left(s_{j}\right)\right|, \quad j=1, \ldots, 1000
$$

Condition number of the corresponding matrix to the system (1.25) is denoted by $\kappa(\mathrm{A})$.

Example 1. Consider the following linear Fredholm integral equation of the second kind

$$
3 \phi(s)+\int_{0}^{1} e^{2 s-\frac{5}{3} \tau} \phi(\tau) d \tau=f(s), \quad 0 \leq s \leq 1
$$

where the function $f(s)$ has been so chosen that $\phi(s)=e^{2 s}$.

Example 2. Consider the following linear Fredholm integral equation of the second kind

$$
\phi(s)-\int_{-1}^{1}\left(1-\tanh ^{2}(\tau)\right) \tanh (s) \tanh (\tau) \phi(\tau) d \tau=f(s), \quad-1 \leq s \leq 1
$$

Here, $f(s)$ is given such that the exact solution is $\phi(s)=e^{-\tanh ^{2}(s)}$.

Table1. Piecewise quadratic interpolation for (1.33) and (1.34)

\begin{tabular}{|c|c|c|c|c|c|}
\hline \multicolumn{3}{|c|}{ Example 1 } & \multicolumn{3}{c|}{ Example 2 } \\
\hline Knots & $E_{\max }$ & $\kappa(\mathrm{A})$ & Knots & $E_{\max }$ & $\kappa(\mathrm{A})$ \\
\hline 5 & $5.61 \mathrm{E}-07$ & 1.97 & 5 & 0.00 & 1.47 \\
\hline 11 & $1.44 \mathrm{E}-08$ & 1.86 & 11 & $1.11 \mathrm{E}-16$ & 1.36 \\
\hline 21 & $8.98 \mathrm{E}-10$ & 1.82 & 21 & $1.11 \mathrm{E}-16$ & 1.34 \\
\hline 41 & $5.61 \mathrm{E}-11$ & 1.80 & 41 & $1.11 \mathrm{E}-16$ & 1.33 \\
\hline 81 & $3.51 \mathrm{E}-12$ & 1.79 & 61 & $1.11 \mathrm{E}-16$ & 1.33 \\
\hline
\end{tabular}

\section{CONCLUSION}

In this paper, a new efficient algorithm for approximating a solution of Fredholm integral equation of the second kind by employing piecewise quadratic approximation with Newton's divided differences approach was presented. A convergence analysis for the method is also provided and tested. As shown in Table1, with respect to the numerical results corresponding to Example 1, it is seen that the absolute error and the condition number of the collocation matrix (1.25) decays when the number of knots, respectively, the size of the matrix increases. However, with Example 2, it is seen that the numerical method is exact and the corresponding errors seen in Table 1 is the half of the unit round-off of 2.2E-16. Accordingly, the proposed method provides a good efficiency.

Moreover, under some reasonable conditions of smoothness and integrability on the kernel, weakly singular integral equations can be also solved using this method. 


\section{REFERENCES}

[1] P. M. Anselone, Collectively Compact Operator Approximation Theory and Applications to Integral Equations, Prentice-Hall, Englewood Cliffs, 1971.

[2] P. M. Anselone and J. W. Lee, Double Approximation Methods for the Solution of Fredholm Integral Equations, ISNM, 30, pp.09-34, 1975.

[3] P. M. Anselone and J. M. Gonzalez-Fernandez, Uniformly Convergent Approximate Solutions of Fredholm Integral equations, J. Math. Anal. App, 10, pp. 519-536, 1965.

[4] K. E. Atkinson, The numerical Solution of Integral Equations of the Second Kind, Cambridge University Press, Cambridge, 1997.

[5] H. Hochstadt, Integral Equations, Wiley Interscience, New York, 1989.

[6] Ram. P. Kanwal, Linear integral equations, theory and technique, Academic Press, INC, New York, 1971.

[7] P. K. Kythe and P. Puri, Computational Methods for Linear Integral Equations, Birkhäuser, Boston, 2002.

[8] A. Rahmoune and M. Nadir, Solving linear Fredholm integral equations of the second kind using Newton divided difference interpolation polynomial, IJMC, 07, pp. 1-6, 2010.

[9] M. Nadir and A. Rahmoune, Modified method for solving linear Volterra integral equations of the second kind using Simpson's rule, IJMM, 1, pp. 133-140, 2007.

[10] Y. Ren, Bo. Zhang, H. Qiao, A simple Taylor-series expansion method for a class of second integral equations, J. Comput. Appl. Math, 110, pp. 15-24, 1999.

[11] D. Porter and DS. G. Stirling, Integral equations, A practical treatment, from spectral theory to applications, Cambridge University Press, Cambridge, 1990.

[12] L. Yucheng, Application of the Chebyshev polynomial in solving Fredholm integral equations, Math. Comput. Modelling, 50, pp. 465-469, 2009. 